\title{
Tensile strength of granular aggregates: Stress chains across particle phase versus stress concentration by pores
}

\author{
Xavier Frank $\odot{ }^{1,}{ }^{, *}$ Farhang Radjaï $\odot,{ }^{2}$ Saied Nezamabadi $\odot,{ }^{2,1}$ and Jean-Yves Delenne ${ }^{1}{ }^{1}$ \\ ${ }^{1}$ IATE, INRAE, Montpellier SupAgro, Université de Montpellier, F-34060, Montpellier, France \\ ${ }^{2}$ LMGC, Université de Montpellier, CNRS, Montpellier, France
}

(Received 12 May 2020; accepted 28 July 2020; published 20 August 2020)

\begin{abstract}
We use the bond-based peridynamics approach to analyze the strength and fracture of dense granular aggregates with variable amount of a solid binding matrix, distributed according to a simple protocol in the interstitial space between particles. We show the versatility of the peridynamics approach in application to crack propagation and its scaling behavior in a homogeneous medium (in the absence of particles and pores). Then we apply this method to simulate the deformation and failure of aggregates as a function of the amount of the binding matrix under tensile loading. We find that the tensile strength is a strongly nonlinear function of the matrix volume fraction. It first increases slowly and levels off as the gap space in-between touching particles is gradually filled by the binding matrix, up to nearly $90 \%$ of the total pore volume, and then a rapid increase occurs to the maximum strength as the remaining interstitial space, composed of isolated pores between four or more particles, is filled. By analyzing the probability density functions of stresses in the particle and matrix phases, we show that the adhesion of the matrix to the particles and the thickening of stress chains (i.e., stresses distributed over larger cross sections) control the strength in the first case whereas the homogenizing effect of the matrix by filling the pores (hence reducing stress concentration) is at the origin of further increase of the strength in the second case. Interestingly, these two mechanisms contribute almost equally to the total strength.
\end{abstract}

DOI: 10.1103/PhysRevE.102.022906

\section{INTRODUCTION}

Porous materials are classically described as a homogeneous matrix hosting connected or disconnected (open) pores and associated with upscaling models essentially based on single-pore or single-crack analysis $[1,2]$. However, this description does not cover the class of porous materials in which porosity is an intrinsic property of their basic microstructure defined by a dense assembly of elementary constituents. Granular aggregates partially filled with a binding matrix belong to this broad class of structured porous materials. It includes concrete $[3,4]$, sintered powders $[5,6]$, sedimentary rocks [7], and wheat endosperm (composed of starch granules distributed in a protein matrix) [8]. Understanding the origins of their elastic and failure properties is of practical interest for the design of concretes [9-13], dense particle-filled composites [14], and other new materials [15-17], as well as for predicting rock fracture $[18,19]$ and the influence of operating parameters on cereal milling $[8,20]$.

Since these granular aggregates have a rich microstructure, their mechanical properties depend not only on the phase volume fractions but also on the geometrical and topological disorder (connectivity and interactions of the particles) of the particle phase [21]. For example, the strength and failure of cemented granular materials and particle-enriched composites have been shown to depend on the adhesion of the inclusions

\footnotetext{
*xavier.frank@inra.fr
}

to the matrix [22,23], as well as the size [22] and shapes of the inclusions $[24,25]$.

In this paper we use peridynamics simulations to quantify and distinguish two different mechanisms underlying the tensile strength of granular aggregates: (1) stress concentration by tensile stress chains encompassing the particles and the binding matrix filling the gap space in between particles, and (2) stress concentration by isolated pores enclosed between several particles. The first mechanism is well known for confined granular materials in which the stress is transmitted along compressive force chains [26]. The second mechanism is common to all porous materials in which stress concentration by the pores controls the strength and toughness of the material [27]. The peridynamics method allows us to discretize both the particles and the matrix, and to follow the evolution of the stress field throughout the samples. We also use a protocol for the distribution of the matrix inside our numerical granular samples so that the interparticle gaps are filled before the remaining isolated pores begin to be filled as the matrix volume fraction is gradually increased. As we shall see, this procedure provides a neat distinction between the two effects.

In the following we first describe the mathematical background of the bond-based peridynamics method and mesh convergence in Sec. II. In Sec. III we present the method used to build the samples. Then in Sec. IV we consider the tensile strength as a function of the matrix volume fraction. In Sec. V we investigate the stress transmission to analyze the two mechanisms underlying the tensile strength. We conclude with a brief discussion of the results and future lines of research. 


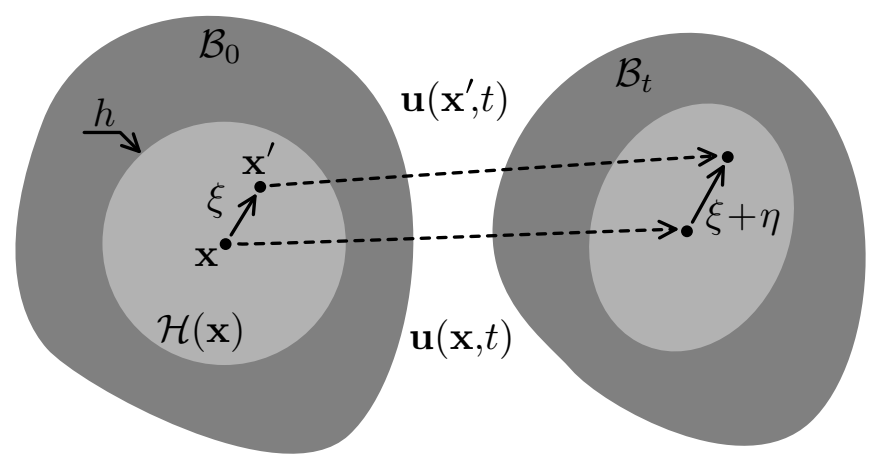

FIG. 1. Left: Reference configuration $\mathcal{B}_{0}$ of a bond $\xi$ connected to a material point $\boldsymbol{x}$ and its horizon domain $\mathcal{H}(\boldsymbol{x})$. Right: Deformed configuration $\mathcal{B}_{t}$. Representation of displacement field $\boldsymbol{u}$ and relative displacement field $\eta$.

\section{NUMERICAL METHOD}

The numerical simulation of the deformation and fracture of inhomogeneous materials is an active field of research $[28,29]$. We used the peridynamics method for its capacity of crack nucleation and computational performance [30,31]. In this method the material is represented as a network of interacting nodes governed by a nonlocal (integral) formulation of the equations of dynamics inside a given "horizon." Bond rupture events lead to damage or to fracture when a critical number of bonds is damaged. Before its application to granular aggregates, we provide in this section a brief description of its principles and show that for brittle materials considered in this paper, this method is accurate and consistent with fracture mechanics. These aspects are important for the validity and robustness of our results.

\section{A. Bond-based peridynamics}

The bond-based peridynamics method is a subset of general state-based peridynamics approaches [32]. This method has been successfully used in a wide range of applications such as the modeling of concrete failure [4], dynamic crack branching [33,34], polycrystal fracture [35], nanoscale rupture mechanics [36,37], microelectronics materials damage [38], and wood failure [39].

Let us consider a domain $\mathcal{B}_{0}$ in $\mathbb{R}^{D}$, where $D$ is space dimension. This domain represents a body in the reference configuration at time $t=0$. The equation of motion for a material point $\mathbf{x} \in \mathcal{B}_{0}$ is given by

$$
\begin{aligned}
\rho(\boldsymbol{x}) \ddot{\boldsymbol{u}}(\boldsymbol{x}, t)= & \int_{\mathcal{H}(\boldsymbol{x})} \boldsymbol{f}\left[\boldsymbol{u}\left(\boldsymbol{x}^{\prime}, t\right)-\boldsymbol{u}(\boldsymbol{x}, t), \boldsymbol{x}^{\prime}-\boldsymbol{x}, \boldsymbol{x}\right] d V_{\boldsymbol{x}^{\prime}} \\
& +\boldsymbol{b}(\boldsymbol{x}, t),
\end{aligned}
$$

where $\rho, \boldsymbol{u}$, and $\boldsymbol{b}$ denote the density, displacement field, and body force, respectively. All material points originally at $\boldsymbol{x}$ in $\mathcal{B}_{0}$ are at the position $\boldsymbol{x}+\boldsymbol{u}$ in $\mathcal{B}_{t}$ at time $t$. The pairwise force exerted between two material points $\boldsymbol{x}$ and $\boldsymbol{x}^{\prime}$ is denoted by $f$. The relative position vector, called bond here, is $\boldsymbol{\xi}=\boldsymbol{x}^{\prime}-\boldsymbol{x} . \mathcal{H}(\boldsymbol{x})$ represents the set of bonds connected to $\boldsymbol{x}$ with lengths below a cutoff distance $h$ called horizon: $\mathcal{H}(\boldsymbol{x})=\left\{\boldsymbol{\xi} \in \mathbb{R}^{D} \mid \boldsymbol{x}+\boldsymbol{\xi} \in \mathcal{B}_{0} \wedge\|\boldsymbol{\xi}\|<h\right\}$ (Fig. 1).
In the most general implementation of peridynamics, called nonordinary state-based models, the force density $\boldsymbol{f}\left[\boldsymbol{u}\left(\boldsymbol{x}^{\prime}, t\right)-\boldsymbol{u}(\boldsymbol{x}, t), \boldsymbol{x}^{\prime}-\boldsymbol{x}, \boldsymbol{x}\right]$ exerted from $\boldsymbol{x}$ on $\boldsymbol{x}^{\prime}$ and $\boldsymbol{f}\left[\boldsymbol{u}(\boldsymbol{x}, t)-\boldsymbol{u}\left(\boldsymbol{x}^{\prime}, t\right), \boldsymbol{x}-\boldsymbol{x}^{\prime}, \boldsymbol{x}^{\prime}\right]$ exerted from $\boldsymbol{x}^{\prime}$ on $\boldsymbol{x}$ can have different directions and magnitudes [32]. In ordinary statebased models, these two forces are supposed to be radial but may have different magnitudes. Here, we choose a bondbased peridynamics approach which neglects the local tangential and torque components: $\boldsymbol{f}\left[\boldsymbol{u}\left(\boldsymbol{x}^{\prime}, t\right)-\boldsymbol{u}(\boldsymbol{x}, t), \boldsymbol{x}^{\prime}-\boldsymbol{x}, \boldsymbol{x}\right]=$ $-\boldsymbol{f}\left[\boldsymbol{u}(\boldsymbol{x}, t)-\boldsymbol{u}\left(\boldsymbol{x}^{\prime}, t\right), \boldsymbol{x}-\boldsymbol{x}^{\prime}, \boldsymbol{x}^{\prime}\right]$. Hence the equation of motion (1) can be simplified by assuming that each bond $\xi$ evolves to a deformed state $\boldsymbol{\xi}+\boldsymbol{\eta}$, where $\boldsymbol{\eta}=\boldsymbol{u}\left(\boldsymbol{x}^{\prime}, t\right)-$ $\boldsymbol{u}(\boldsymbol{x}, t)$ is the relative displacement between the bond endpoints:

$$
\rho(\boldsymbol{x}) \ddot{\boldsymbol{u}}(\boldsymbol{x}, t)=\int_{\mathcal{H}(\boldsymbol{x})} \boldsymbol{f}(\boldsymbol{\xi}, \boldsymbol{\eta}, \boldsymbol{x}) d V_{\boldsymbol{x}^{\prime}}+\boldsymbol{b}(\boldsymbol{x}, t) .
$$

Thus a pairwise force $f$ can be defined from the bond initial state $\boldsymbol{\xi}$ and its relative displacement $\eta$ :

$$
\boldsymbol{f}(\boldsymbol{\xi}, \boldsymbol{\eta})=f(\boldsymbol{\xi}, \boldsymbol{\eta}, \boldsymbol{x}) \frac{\boldsymbol{\xi}+\boldsymbol{\eta}}{\|\boldsymbol{\xi}+\boldsymbol{\eta}\|},
$$

where $f(\boldsymbol{\xi}, \boldsymbol{\eta}, \boldsymbol{x})$ is a scalar representing the magnitude of the force in units of force per unit volume squared. Inserting (3) into the equation of motion (2) yields

$$
\rho(\boldsymbol{x}) \ddot{\boldsymbol{u}}(\boldsymbol{x}, t)=\int_{\mathcal{H}(\boldsymbol{x})} f(\boldsymbol{\xi}, \boldsymbol{\eta}, \boldsymbol{x}) \frac{\boldsymbol{\xi}+\boldsymbol{\eta}}{\|\boldsymbol{\xi}+\boldsymbol{\eta}\|} d V_{\boldsymbol{x}^{\prime}}+\boldsymbol{b}(\boldsymbol{x}, t) .
$$

In this paper, inhomogeneous elastic materials are investigated where the force density $\boldsymbol{f}$ depends on different phases. Hence the elasticity of each bond $\xi$ explicitly depends on both points $\boldsymbol{x}$ and $\boldsymbol{x}+\boldsymbol{\xi}$. We assume a harmonic potential energy for which the force function is given by

$$
f(\boldsymbol{\xi}, \boldsymbol{\eta}, \boldsymbol{x})= \begin{cases}c_{\boldsymbol{x}, \boldsymbol{x}+\boldsymbol{\xi}} s(\boldsymbol{\xi}, \boldsymbol{\eta}) & \|\boldsymbol{\xi}\| \leqslant h \\ 0 & \|\boldsymbol{\xi}\|>h\end{cases}
$$

where $s$ is the bond elongation

$$
s(\boldsymbol{\xi}, \boldsymbol{\eta})=\frac{\|\boldsymbol{\xi}+\boldsymbol{\eta}\|-\|\boldsymbol{\xi}\|}{\|\boldsymbol{\xi}\|}
$$

and $c_{x, x+\xi}$ is the so-called "elastic micro-modulus" and $c_{\boldsymbol{x}+\boldsymbol{\xi}, \boldsymbol{x}}=c_{\boldsymbol{x}, \boldsymbol{x}+\boldsymbol{\xi}}$. For all points located in the pores or outside the domain we set $c=0$. The effective Young's modulus of a homogeneous sample is given by [34]

$$
E=\frac{c \pi h^{3}(1-v)}{6}
$$

which linearly depends on $c$. The Poisson's ratio is $v=1 / 3$ for the bond-based peridynamic approach in two dimensions (2D) [4].

To take into account the possibility of fracture, we assume that a bond fails when it exceeds its critical elongation $s_{0}$, which depends on the phase (Fig. 2). The fracture energy $G_{c}$ of a homogeneous material of elastic modulus $E$ and critical elongation $s_{0}$ is given by [34]

$$
G_{c}=\frac{9 E h s_{0}^{2}}{4 \pi}
$$




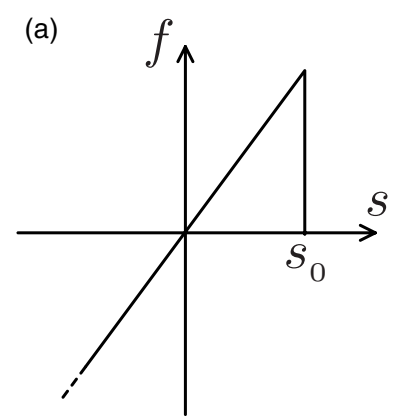

(b)

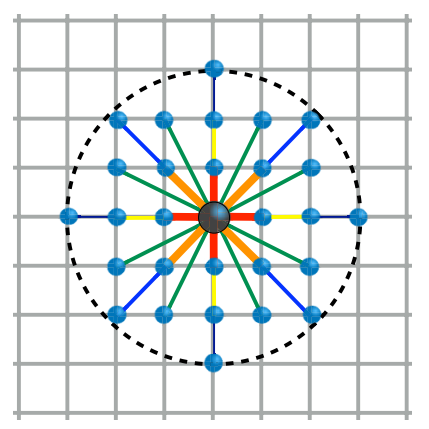

FIG. 2. (a) Schematic representation of the pairwise force $f$ as a function of bond elongation $s$. Failure can occur only in tension for a critical elongation $s_{0}$. (b) Connectivity of bonds for a horizon of 3 .

Furthermore, the toughness $K$ of the material is related to $G_{c}$ and $E$ through $K=\sqrt{E G_{c}}$.

In peridynamics approaches, both cracks and interfaces between phases have a finite thickness depending upon $h / \delta x$. To overcome this blurring effect, higher spatial resolutions are needed. Another drawback of the bond-based peridynamic approach is that the mechanical behavior is brittle. An arbitrary mechanical behavior may be implemented by means of a state-based peridynamic approach, but it involves larger computing time. Despite the simplicity of the bond-based peridynamic approach, it has the advantage of allowing for complex material features to be implemented in this framework, such as anisotropic fabric or a fiber-reinforced matrix [40-42]. In this paper we focus on highly heterogeneous distributions of the phases.

Most studies using the peridynamics approach have concerned the dynamic propagation of cracks $[31,33,34]$ and dynamic fragmentation [31]. In these studies an explicit time integration scheme is generally used. Much less attention has been paid to quasistatic conditions, which are of great interest for low-rate processes $[43,44]$. Several techniques have been reported in the literature for quasistatic simulations in the context of lattice approaches [23]. In most cases, the sample is loaded until one bond reaches a critical stress (or elongation). This critical bond is then removed and, after a full relaxation of the system, the load is incremented. The main disadvantage of this procedure is its high computational cost. For this reason, we choose here to allow several bonds to break during each load increment. See Appendix A for further details.

\section{B. Discretization}

The simulated domain is discretized into a twodimensional $N_{x} \times N_{y}$ rectilinear grid of spatial resolution $\delta x$. Each material point $i$ of position $\boldsymbol{x}_{i}$ has a mass $m_{i}=$ $(\delta x)^{2} \rho\left(\boldsymbol{x}_{i}\right)$. The system can be viewed as a mass-spring lattice in which each bond (linear spring) connects two points on the grid. The equation of motion (4) is discretized on the grid:

$$
m_{i} \ddot{\boldsymbol{u}}_{i}(t)=\sum_{\boldsymbol{x}_{j} \in \mathcal{H}\left(\boldsymbol{x}_{i}\right)} k_{i j} s\left(\boldsymbol{\xi}_{i j}, \boldsymbol{\eta}_{i j}\right) \frac{\boldsymbol{\xi}_{i j}+\boldsymbol{\eta}_{i j}}{\left\|\boldsymbol{\xi}_{i j}+\boldsymbol{\eta}_{i j}\right\|}+\boldsymbol{b}_{i}(t),
$$

where $\boldsymbol{u}_{i}(t)=\boldsymbol{u}\left(\boldsymbol{x}_{i}, t\right), \boldsymbol{\xi}_{i j}=\boldsymbol{x}_{j}-\boldsymbol{x}_{i}, \boldsymbol{\eta}_{i j}=\boldsymbol{u}_{j}-\boldsymbol{u}_{i}, \boldsymbol{b}_{i}(t)=$ $(\delta x)^{2} \boldsymbol{b}\left(\boldsymbol{x}_{i}, t\right)$, and $k=c(\delta x)^{4}$. Since no excluded-volume constraints are imposed on the points, the strain should be kept small enough to avoid overlaps between neighboring bonds and ensure a macroscopic linear behavior.

The stress tensor at each node $i$ is given by the following equation [45]:

$$
\sigma_{i}(t)=\frac{1}{\delta_{x}^{2}} \sum_{\boldsymbol{x}_{j} \in \mathcal{H}\left(\boldsymbol{x}_{i}\right)} \boldsymbol{f}_{i j} \otimes\left(\boldsymbol{\xi}_{i j}+\boldsymbol{\eta}_{i j}\right),
$$

where $f_{i j}$ is the force exerted from material point $i$ to material point $j$.

The number of bonds interacting with each grid point depends on $h / \delta_{x}$. Figure 2(b) shows an example of bond connectivity for a horizon $h=3 \delta_{x}$. The node connectivity $W_{p}$ increases with $h / \delta_{x}$ approximately as a power law of exponent 2. For example, for $h / \delta_{x}$ varying from 1 to 6 , we have $W_{p}=4$, $12,28,48,80$, and 112, respectively. Finally, it is interesting to note that, although the determination of the number of points on a regular lattice within a given distance is numerically straightforward, it is not fully resolved analytically. This problem is known as the Gauss circle problem and remains an open mathematical problem [46]. We developed a parallel implementation of the discretized bond-based peridynamics approach with the help of a message passing interface (MPI). The simulation domain was split into a regular grid of subdomains, each one being attributed to a single CPU core. See Appendix B for further details about the scaling behavior of the code.

\section{Numerical accuracy}

The accuracy of a mesh-based simulation depends on the numerical method, type of elements, and the way the simulated domain is discretized. One needs to find a tradeoff between numerical accuracy and computation time. As finer meshes generally lead to a more accurate solution but longer computation time, it is necessary to quantify the required accuracy for the problem at hand and the mesh effects with respect to the considered behavior, such as failure stress or toughness. Below, we will show that the global Griffith fracture approach is consistent with our simulations.

In peridynamics approaches, both the density of the mesh (which depends on the number of elements in $x$ direction $N_{x}$ ) and the resolution of the horizon (which depends on $h / \delta x$ ) should be fixed. For an inhomogeneous material, the rectilinear mesh leads to the rasterization of the interfaces but also to a blurring effect due to the nonlocal interactions. This issue was extensively studied in [47,48], and the authors concluded that a horizon of 2 should be avoided, but a horizon of 3 , which corresponds to 28 bonds per node, provides a good compromise between the accuracy of the solution and computational efficiency. We studied mesh convergence of elasticity properties, and our results confirmed their conclusion.

The failure stress or the strength $\sigma_{c}$ of a homogeneous material can be deduced from the Young's modulus $E$ and critical elongation $s_{0}$ as $\sigma_{c}=E s_{0}$. In multiphase or damaged materials, failure is triggered by stress concentration in the vicinity of defects, and the failure is described in terms of both the strength $\sigma_{c}$ and toughness $K$ of the material [49-51]. According to the classical fracture mechanics, for a single crack of length $2 a$, the toughness in mode-I fracture is given 


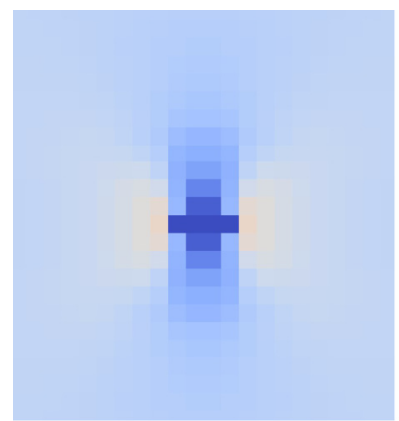

(a)

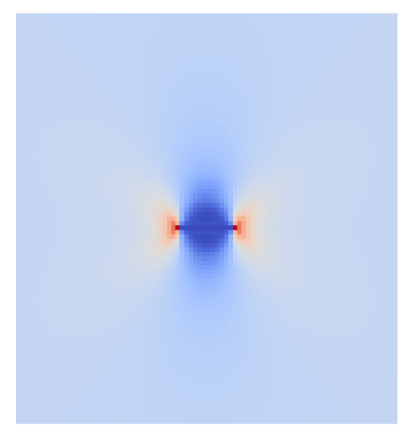

(c)

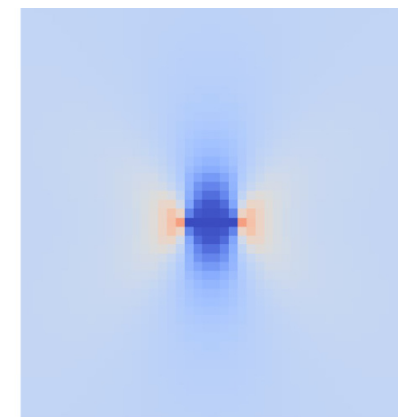

(b)

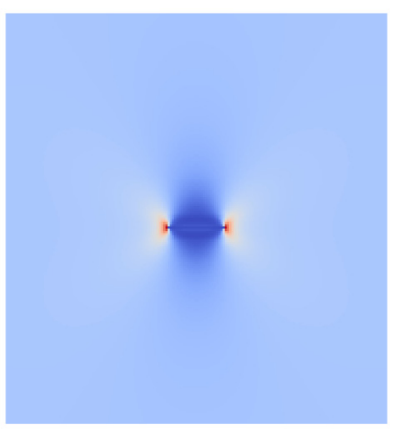

(d)
FIG. 3. Stress field around a crack before failure for $h / \delta_{x}=6$, $a=0.0125$ and (a) $N_{x}=128$, (b) $N_{x}=256$, (c) $N_{x}=512$, and (d) $N_{x}=1024$.

by

$$
K=\sigma_{c} \sqrt{\pi a}=\sqrt{E G_{c}},
$$

where $G_{c}$ is the critical energy release rate. Hence the ratio $\sigma_{c} / K$ as a function of $1 / \sqrt{\pi a}$ is expected to be a linear function of slope 1. In the same way, setting that the theoretical toughness is $K_{t h}=\sqrt{E G_{c}}$, the ratio $\sigma_{c} / K_{t h}$ as a function of $1 / \sqrt{\pi a}$ should be a linear function of slope $K / K_{t h}$, where $K=$ $\sigma_{c} \sqrt{\pi a}$ is the value of toughness measured from peridynamics simulations.

In the case of a sufficiently small defect, knowing that $\sigma_{c}=$ $E s_{0}$, a length $l_{p}$ can be defined from Eqs. (8) and (11):

$$
l_{p}=\frac{2}{\pi}\left(\frac{K}{\sigma_{c}}\right)^{2}=\frac{9}{2 \pi^{2}} h .
$$

This length represents that of the fracture process zone (FPZ), where the material undergoes damage ahead of the fracture tip. The above expression indicates that the horizon $h$ is more than a purely numerical parameter and can be physically interpreted as the size of FPZ.

To investigate the evolution of $K$ as a function of both spatial resolution and horizon and for different values of the crack length, we performed extensive simulations of a homogeneous sample containing a single central crack perpendicular to the direction of extension, as shown in Fig. 3. The sample is square-shaped and its side length is 1 . The initial crack is a segment, and the bonds crossing it are broken at time $t=0$. The simulations were carried out for a set of values of $h / \delta x=$ $2,3,4,5,6,7,8$ and $N_{x}=128,256,512,665,1024$, and in each case the crack half-length was set to different values

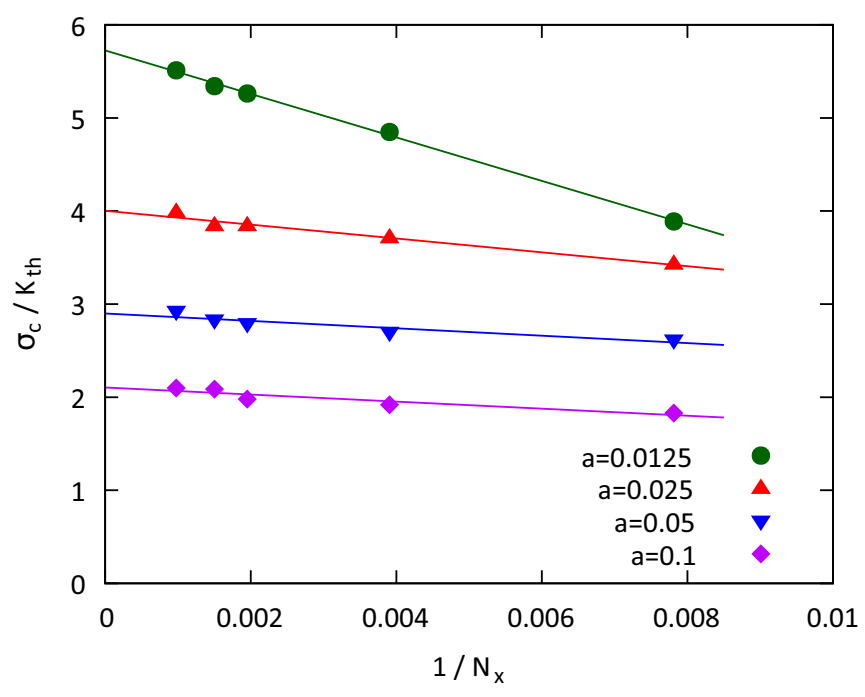

FIG. 4. Failure stress $\sigma_{c}$ of a homogeneous material including a single crack of length $2 a$ as a function of $1 / N_{x}$ for $h / \delta x=3$ and several values of $a . \sigma_{c}$ is normalized by the theoretical toughness $K_{t h}=\sqrt{E G_{c}}$. The straight lines are a linear regression to the data points.

$a=0.1,0.05,0.025,0.0125$. With these sets of parameters, 140 simulations were performed.

Let us first consider the spatial mesh convergence at a fixed value of $h / \delta x$. Figure 4 displays $\sigma_{c} / K_{t h}$ as a function of $1 / N_{x}$ for different values of $a$ in the case $h / \delta x=3$. For all crack lengths, we observe a nearly linear increase of $\sigma_{c}$ as $N_{x}$ increases, with larger variations and larger strength for shorter crack lengths. The asymptotic value of the strength can be obtained by a linear extrapolation in the limit $1 / N_{x} \rightarrow 0$ (limit of infinite precision). The finite spatial resolution leads to reduced elastic moduli and failure stress due to the insufficient number of elements or meshes to represent the continuum elasticity correctly $[13,52,53]$. This value depends on both $a$ and the horizon $h / \delta x$. Its values are plotted as a function of $1 / \sqrt{\pi a}$ for different values of $h / \delta x$ in Fig. 5. As expected, $\left[\sigma_{c} / K_{t h}\right]_{N_{x} \rightarrow \infty}$ is a linear function of $1 / \sqrt{\pi a}$, and the slope of the curve for a given $h / \delta x$ can be identified as $\left[K / K_{t h}\right]_{N_{x} \rightarrow \infty}$, measured by peridynamics simulations. This slope is plotted in Fig. 6 as a function of $h / \delta x$. Its value is above 1 at small horizon and tends to 1 as the horizon increases.

The above results show how the accuracy of peridynamics simulations increases with $h / \delta_{x}$. However, a large horizon such as $h / \delta_{x}=8$ implies a larger number of interactions and thus long simulations. The choice $h / \delta_{x}=3$ remains a good compromise between numerical efficiency and accuracy. This is consistent with earlier results [54]. Furthermore, the accuracy gained by a larger value of $h / \delta_{x}$ is too expensive and unnecessary to delineate the regime transition addressed in the following.

The examples presented and discussed in this section prove that the peridynamics method captures the continuum-scale quantities such as toughness despite its discrete nature, which removes stress singularities and does not necessarily meet the assumptions of scale separability as required by the continuum mechanics models of fracture. The bond-based 


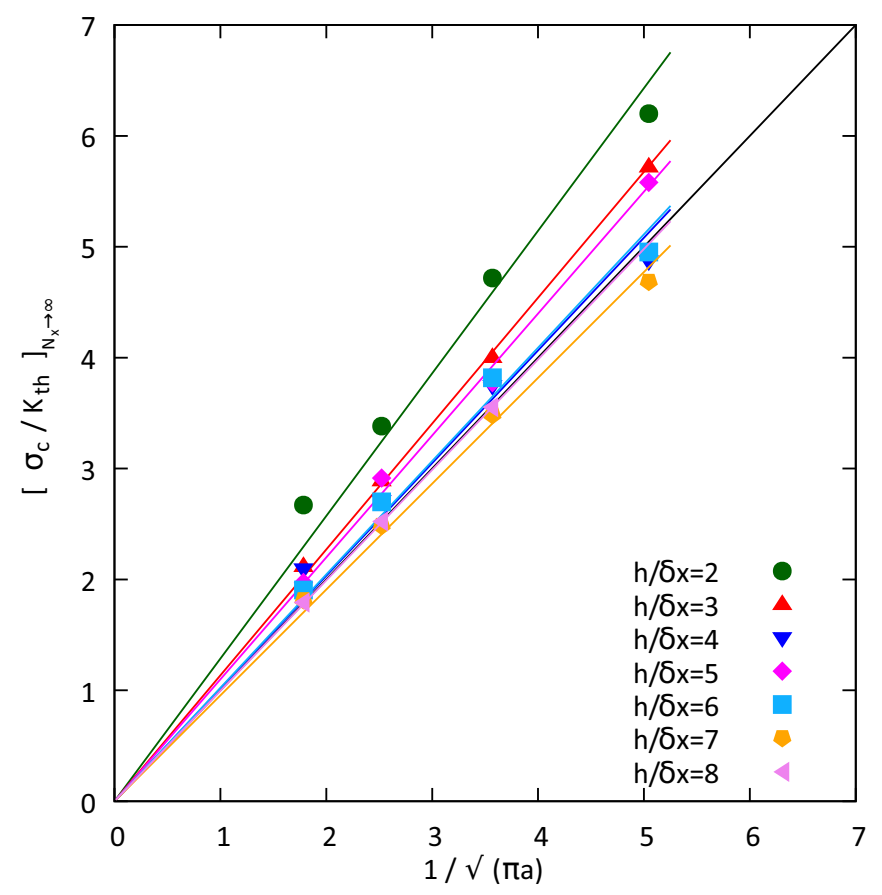

FIG. 5. Extrapolated value of normalized failure stress at infinite resolution in a homogeneous sample that contains a single crack of length $2 a$ as a function of $1 / \sqrt{\pi a}$ for several values of $h / \delta x$. Straight lines represent linear fits to the data. The slopes represent in each case the ratio $\left[K / K_{t h}\right]_{N_{x} \rightarrow \infty}$ in the high-resolution limit.

peridynamics is used here for both its capacity of crack nucleation and propagation and its computational performance and versatility. In its basic formulation, the simulated mechanical behavior is brittle and Poisson's ratio is $1 / 3$. Although this method can be extended for the simulation of other mechanical behaviors, it is fully suited in its basic form to multiphase materials in which the complexity of the mechanical behavior arises from the geometrical texture.

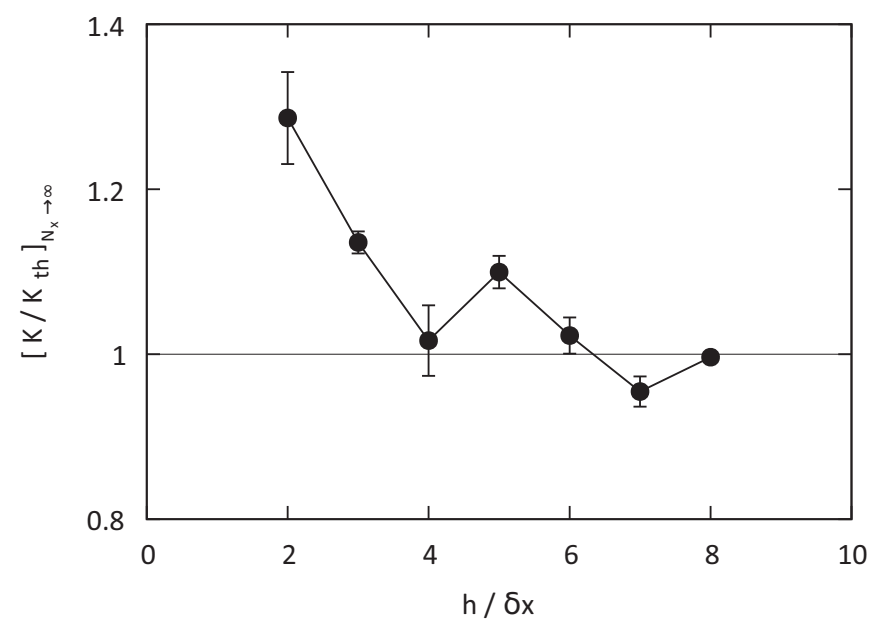

FIG. 6. Infinite-resolution normalized toughness $\left[K / K_{t h}\right]_{N_{x} \rightarrow \infty}$ measured from peridynamics simulations as a function of $h / \delta x$. Error bars represent standard deviation. (a)

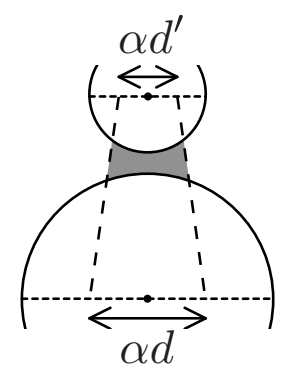

(c)

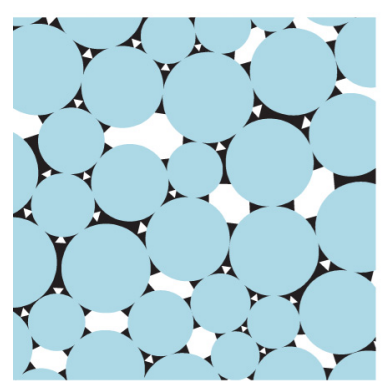

(b)

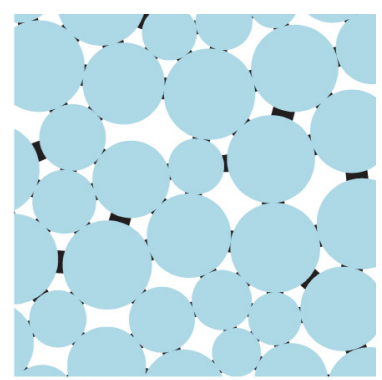

(d)

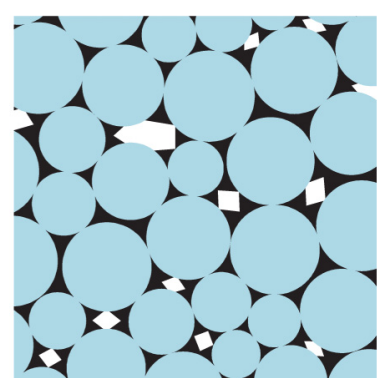

FIG. 7. (a) Algorithm for inserting the binding matrix in the form of pairwise trapezoidal bridges in the gap space in between pairs of neighboring particles. Snapshots for $\alpha=0.25$ (b), $\alpha=0.5$ (c), and $\alpha=0.75$ (d).

\section{SAMPLE BUILDING}

To generate the microstructure with a distribution of nonoverlapping particles, we used the discrete element method (DEM). The particles are polydisperse disks placed initially inside a 2D simulation box of side lengths $L_{x}$ and $L_{z}$. Then a weak confining pressure (compared to particle stiffness) is applied to the walls of the simulation box until a fully jammed state is reached with an aspect ratio $L_{z} \simeq 2 L_{x}$ [45]. The particle volumes have a uniform distribution [55] with a size ratio of 2 between the largest and smallest particles. Due to isotropic compaction, the resulting packing has an isotropic texture in terms of contact orientations and forces. Ten independent samples of 400 particles were prepared using this procedure.

The binding matrix is then added to the interstitial space between particles according to the following protocol. A trapezoidal-shaped bridge of the matrix phase is added in the gap space between all pairs of particles for which the center-to-center distance is below $\beta\left(d+d^{\prime}\right) / 2$, where $d$ and $d^{\prime}$ are the diameters of the paired particles and $\beta$ is a control parameter $[8,56]$. For $\beta=1$, the eligible pairs include only the pairs of touching particles. As illustrated in Fig. 7, the thickness of the bridges is a fraction $\alpha$ of the particle diameters. For sufficiently low values of $\alpha$, to which we will refer as a gapfilling factor, the binding matrix connects only adjacent pairs of particles [Fig. 7(b)]. When $\alpha$ increases, the thickness of the matrix bridge increases as shown in Fig. 7(c), and for $\alpha=1$, the bridge fills nearly the whole gap between the two particles; see Fig. 7(d). At this point, some neighboring bridges overlap. In particular, all the pores enclosed between three particles are filled, and the remaining pore space is composed of isolated pores enclosed between four or more particles. To fill these 


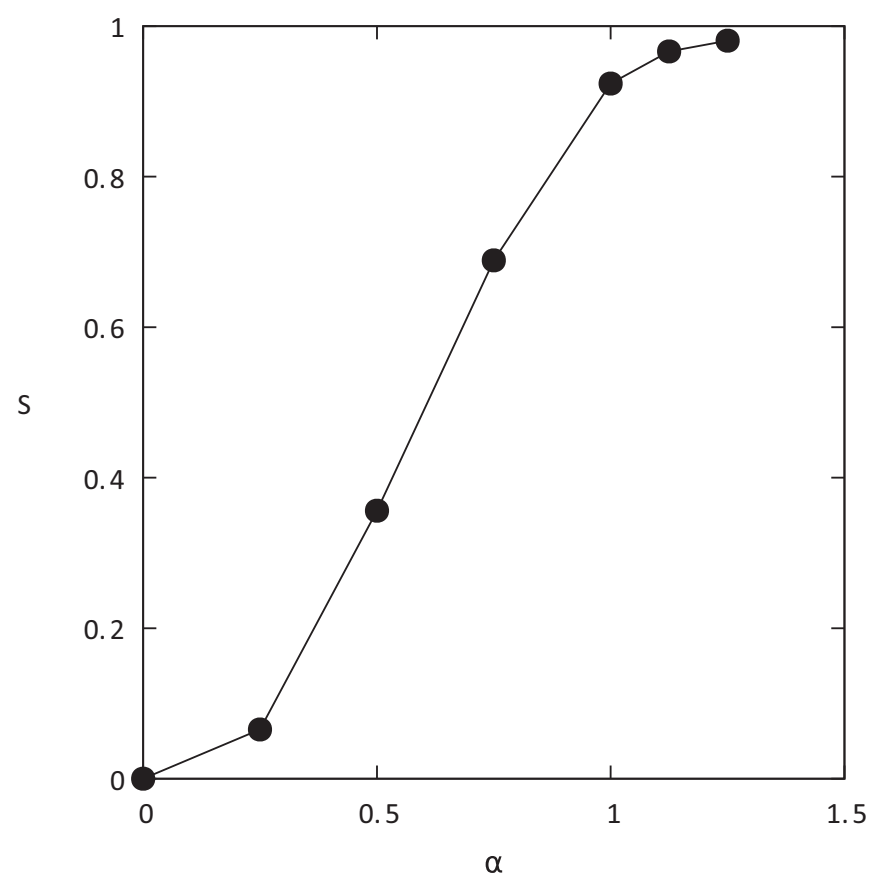

FIG. 8. Saturation factor $S$ of the binding matrix as a function of the gap-filling factor $\alpha$. The data points are averages over ten independent samples. Error bars are smaller than the symbols.

pores, $\alpha$ must further be increased beyond 1 . In the following, we refer to the matrix for $\alpha<1$ as a gap-filling matrix. Of course, other protocols for the distribution of the matrix phase are possible. The protocol used here has the advantage of allowing the gap-filling matrix to be clearly distinguished from the pores of higher order, i.e., pores that do not belong to a gap space in between a pair of particles.

In our simulations, we set $\beta=1.1$ so that the solid bridges are attributed only to the touching and very close pairs of particles. The parameter $\alpha$ was varied from 0.25 to 1.25 . Let $\Phi_{m}$ be the matrix volume fraction and $\Phi_{v}$ the volume fraction of the interstitial space between particles (called also void space). The saturation factor $S=\Phi_{m} /\left(\Phi_{m}+\Phi_{v}\right)$ represents the volume fraction of the void space filled by the matrix. The saturation factor increases with $\alpha$, as shown in Fig. 8. Note that $S$ is a nearly linear function of $\alpha$ for intermediate values of $\alpha$. For $\alpha=1,90 \%$ of the interstitial space is filled. This means that the isolated pores at this filling degree represent only $10 \%$ of the interstitial volume between particles. We also created a sample with $S=1$ by simply filling the whole void space.

Figure 9 displays zoomed snapshots of the pore space for different values of the saturation factor $S$. Both the number and size of the pores decrease by orders of magnitude with increasing $S$. We used a flood fill algorithm to build the list of pores and their volumes in the samples. Figures 10 and 11 show the number and average volume (area in 2D) of the pores, respectively, as a function of $S$. We see that the number of pores declines continuously beyond $S=0.34$, whereas the average pore volume declines significantly only at higher values of $S$ close to 1 , reflecting the filling of isolated pores. The largest pore volume, shown also in Fig. 11, follows a similar trend. (a)

(b)

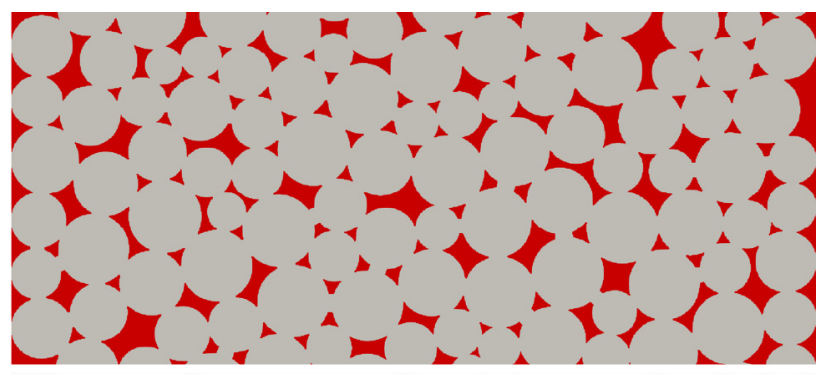

(c)

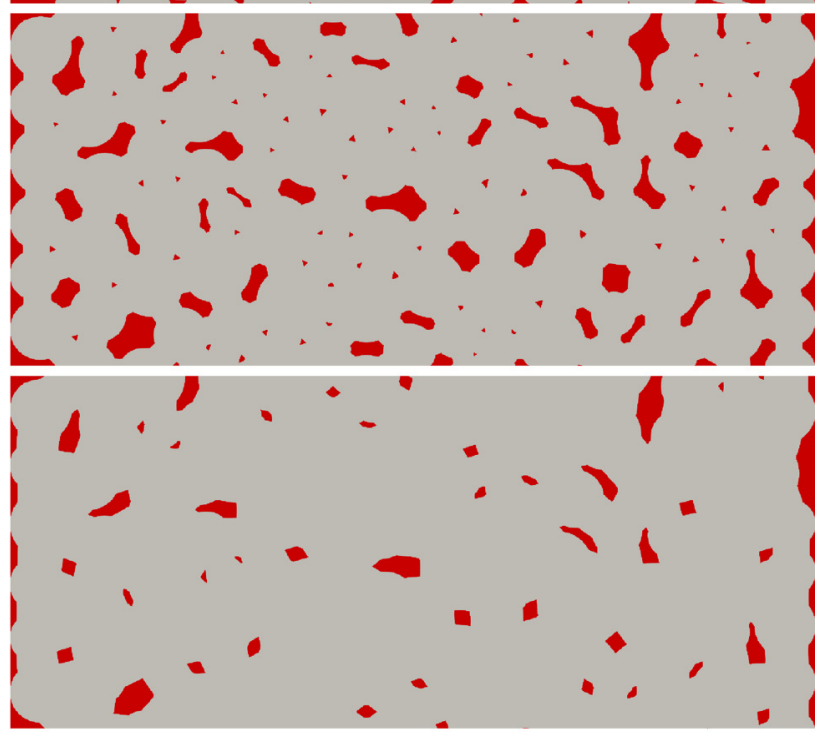

(d)

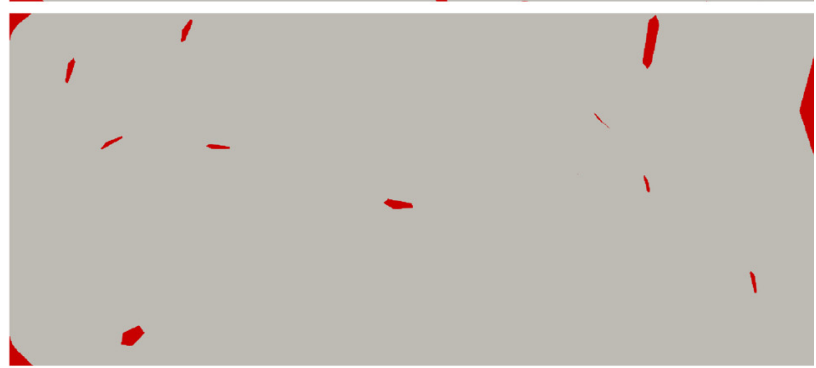

(e)

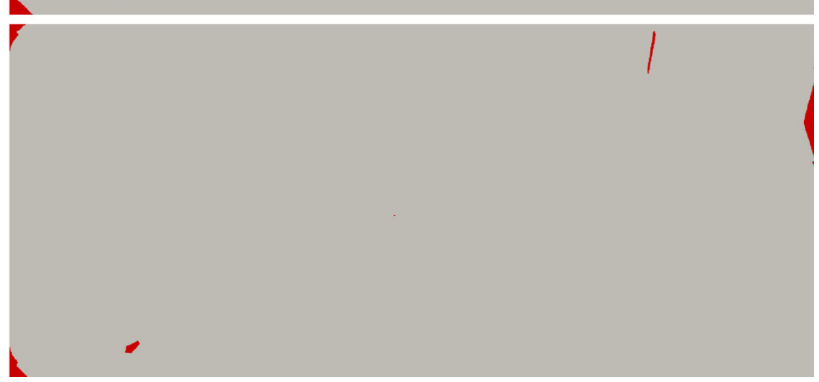

FIG. 9. Pores in cemented granular materials for (a) $S=0.064$, (b) $S=0.34$, (c) $S=0.67$, (d) $S=0.91$, and (e) $S=0.97$.

\section{TENSILE STRENGTH}

The samples created by the compaction of rigid particles in a box and filled with a given amount of matrix are discretized on a lattice. The lateral walls (along the $x$ axis) are removed, and the top and bottom walls are assumed to have the same mechanical properties as the particles. The bottom wall is fixed, and the top wall is subjected to incremental quasistatic extension at constant rate along the $z$ axis. 


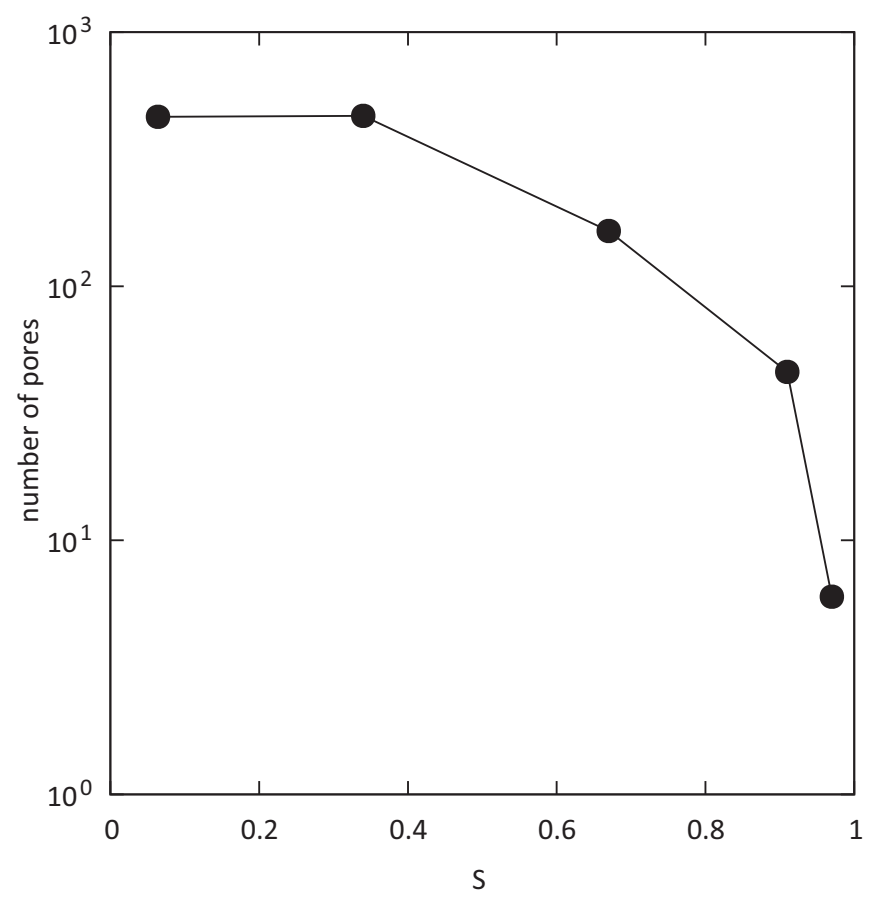

FIG. 10. Number of pores in a sample as a function of the saturation factor $S$.

Each bond connecting two lattice points $i$ and $j$ (closer than the horizon $h$ ) has a stiffness $k_{i j}$ depending on the phase in which the points are located. When $i$ or $j$ is in the pores, $k_{i j}=0$; when $i$ and $j$ are in the matrix, $k_{i j}=k_{M M}$; and when $i$ and $j$ are in the same particle, $k_{i j}=k_{P P}$. Otherwise, $k_{i j}=k_{P M}$ when $i$ and $j$ are in different phases or in different particles.

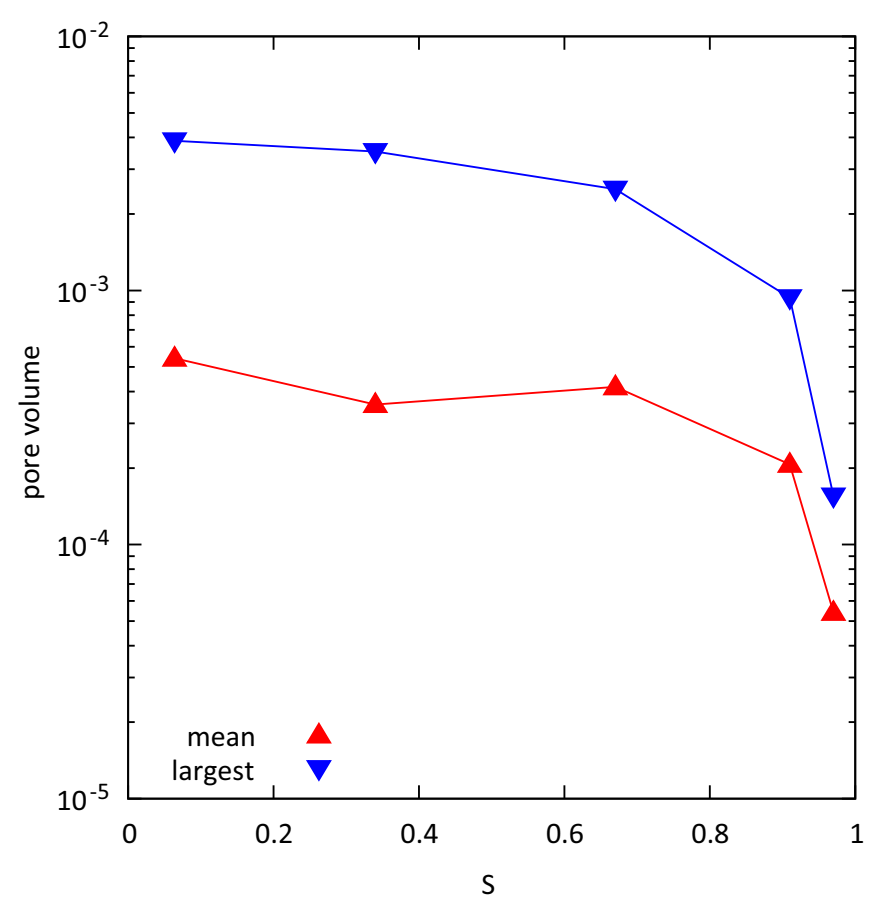

FIG. 11. The average volume of pores and the largest pore volume in a sample as a function of the saturation factor $S$.

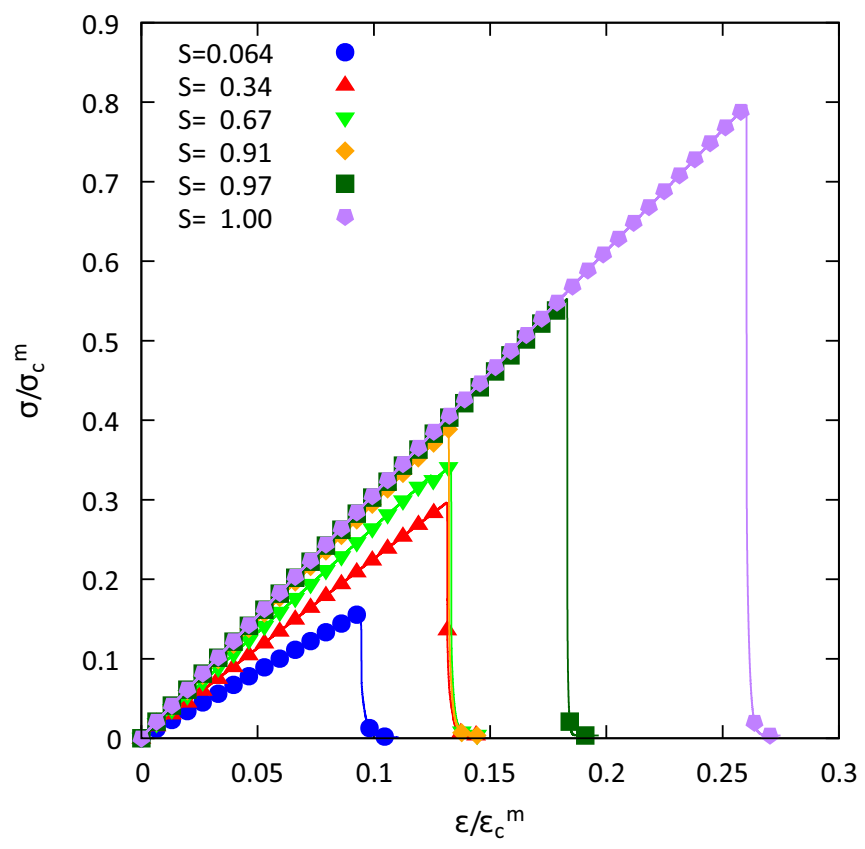

FIG. 12. Stress-strain relationships for different values of $S$. The stress is normalized by the matrix failure stress, and the strain is normalized by the matrix failure strain.

The value of the critical elongation $s_{0 i j}$ follows the same assumptions: when $i$ and $j$ are in the matrix, $s_{0 i j}=s_{0 M M}$; when $i$ and $j$ are in the same particle, $s_{0 i j}=s_{0 P P}$; otherwise, $s_{0 i j}=s_{0 P M}$.

The ratio of Young's modulus $E_{P P}$ of the particles to that of the matrix $E_{M M}$ was set to $\psi=E_{P P} / E_{M M}=4$. This choice implies that most of the deformation occurs in the matrix phase. In the same way, the ratio of the toughness of the particles to that of the matrix was set to $K_{P P} / K_{M M}=\psi=4$. The particle-matrix interface is assumed to have the same properties as the matrix: $E_{P M}=E_{M M}$ and $K_{P M}=K_{M M}$. This choice of the mechanical parameters was motivated by the general observation that in most aggregates the matrix is the weakest phase and the properties of the interface are generally closer to those of the weaker phase. The focus of this paper is on the effect of the binding matrix. According to our earlier results [57], the value $\psi=4$ is high enough to ensure that particles will not be damaged during tensile tests and the cracks propagate only inside the binding matrix. A similar analysis can be performed for other values of the phase material properties.

Figure 12 shows the vertical stress, normalized by the tensile strength $\sigma_{c}^{m}$ of the matrix, as a function of vertical strain $\varepsilon$, normalized by the fracture strain $\varepsilon_{c}^{m}=\sigma_{c}^{m} / E_{M M}$, for different values of $S$. In all cases we observe a brittle linear elastic behavior with an increasing effective Young's modulus and fracture stress as $S$ increases. The tensile strength of the aggregate is the fracture stress $\sigma_{c}$ at the failure point. Up to the failure point, no breakage events are observed inside the sample, which continues to deform elastically. At failure, a crack nucleates and propagates instantly across the sample, leading to sudden stress drop. Figure 13 shows two examples of typical crack paths. The cracks are extended damaged 
(a)

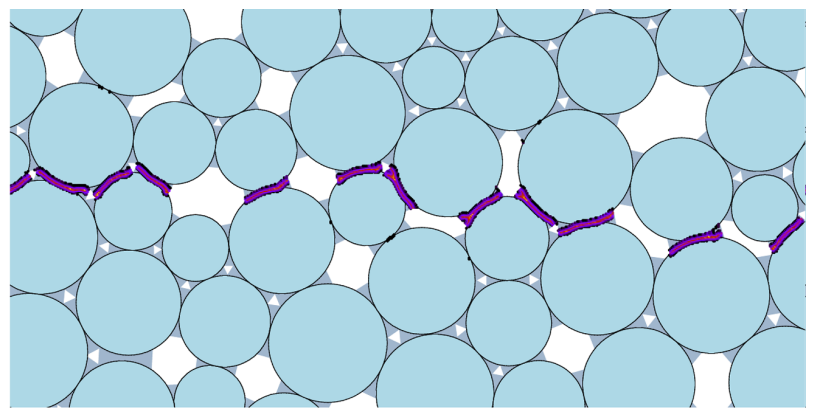

(b)

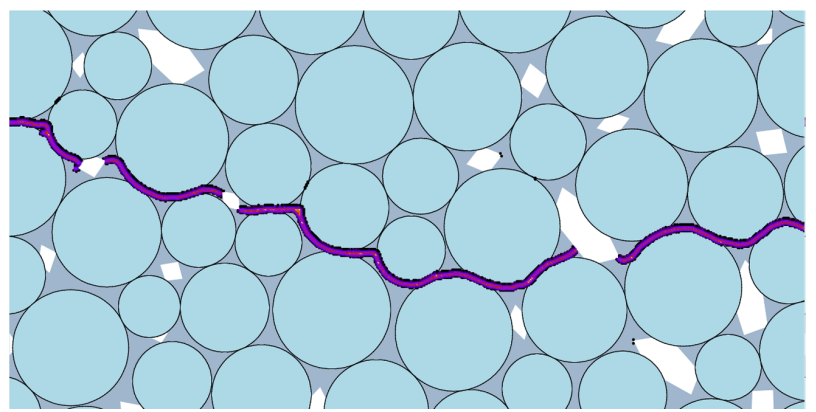

FIG. 13. Zoomed snapshots of crack paths for (a) $S=0.34$ and (b) $S=0.67$.

zones with a constant thickness controlled by the size of the peridynamics horizon. As expected, they are initiated in the matrix and cannot penetrate the particles since the matrix has a lower toughness than the particles $(\psi=4)$. For this reason the tensile strength depends on the strength of the matrix phase and the arrangement of the particles, and may thus be quantified in terms of a tortuosity parameter [58].

Figure 14 shows the tensile strength $\sigma_{c}$ as a function of the saturation factor $S$. The tensile strength first increases rapidly with $S$ in the range from 0 to 0.4 , then increases only slightly from 0.4 to 0.9 before rising strongly again as $S$ increases from 0.9 to 1 . The tensile strength is nearly doubled in this range, increasing from $\sigma_{c} \simeq 0.38 \sigma_{c}^{m}$ to $\sigma_{c} \simeq 0.78 \sigma_{c}^{m}$. This means that for $S=1$, the matrix filling the isolated higher-order voids, representing only $10 \%$ of the total matrix volume, contributes as much to the strength of the aggregate as the gap-filling matrix, corresponding to $90 \%$ of the total matrix volume. This behavior reflects stress concentration by both the particle chains and the isolated pores that we analyze in detail below. In the fully saturated case $S=1$, the ratio $\sigma_{c} / \sigma_{c}^{m} \simeq 0.78$ reflects the fact that the crack propagates only in the matrix phase following a tortuous path.

\section{STRESS TRANSMISSION}

The tensile stress in cemented aggregates is transmitted through both the particle phase and the matrix phase. As the amount of the matrix increases, we expect an increasingly homogeneous transmission of the stress, with the matrix playing two different roles depending on whether it fills the gaps in-between the particles or the remaining isolated pores when the gaps are filled. In other words, as long as the saturation factor $S$ is below 0.9 , we expect the matrix just to bridge the particles, allowing tensile stresses to be transmitted across the particle phase. For larger values of $S$ the higher-order pores

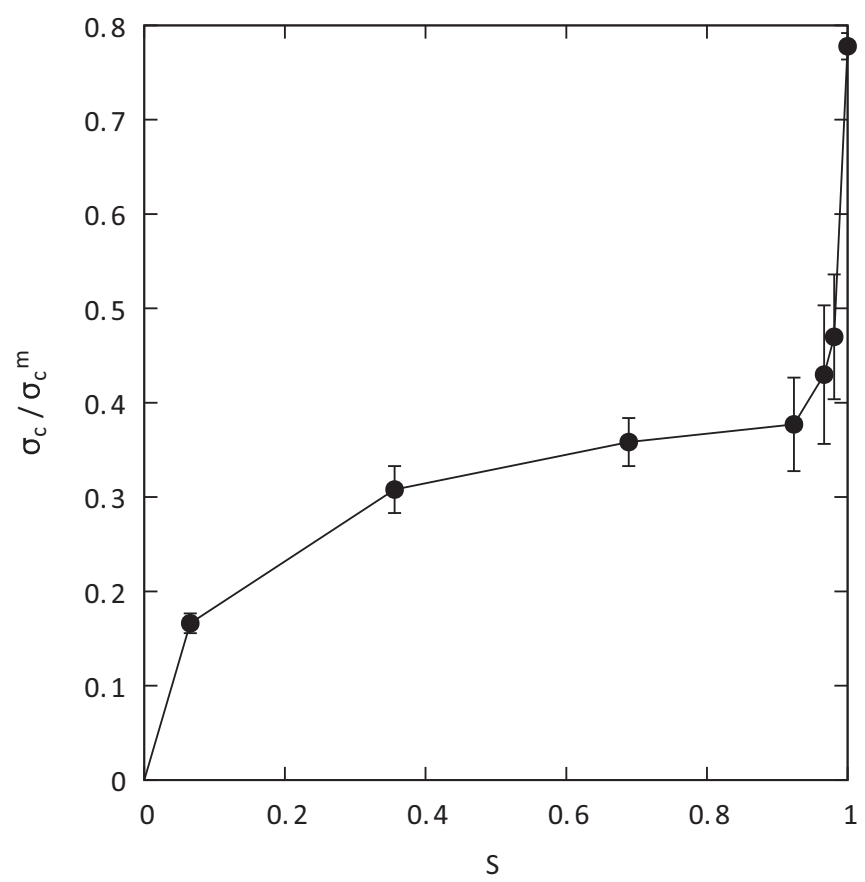

FIG. 14. Tensile strength $\sigma_{c}$ normalized by the strength $\sigma_{c}^{m}$ of the matrix phase as a function of the saturation factor $S$. The data points are averages over ten samples, and the error bars are the corresponding standard deviations.

are filled by the matrix, and thus stress concentration by the pores is reduced. We would like to clarify the signature of these two different effects on the distribution of tensile and compressive stresses inside the material.

Figure 15 displays several zoomed maps of the vertical stress field $\sigma_{z z}$ for different values of $S$ for $\epsilon / \epsilon_{c}{ }^{m}=1.62 \times$ $10^{-2}$. At low values of $S$, the tensile (positive) stresses are concentrated at the contact points between particles, which define stress chains across the contacts and particles throughout the system, although they are blurred inside the particles, showing both large stresses close to the contact points and a large number of vanishing stress values. The stress chains thicken with increasing volume of the matrix, while losing their intensity. At larger values of $S$ we still observe the stress chains, but the patterns of stress concentration are correlated both with the remaining pores and with the particle positions. This is consistent with a recent work showing that stress chains can be induced not only by the contact network but also by the presence of disordered pores inside a continuous matrix [27].

The compressive forces (not shown in Fig. 15) are by at least one order of magnitude lower than tensile forces. The mean values of the tensile (positive) and compressive (negative) vertical stress $\sigma_{z z}$ for $\epsilon / \epsilon_{c}{ }^{m}=1.62 \times 10^{-2}$ are shown in Fig. 16 as a function of $S$. As expected, the average tensile stress increases with $S$. Moreover, its value is well above that in the matrix alone $\left.\left(\left\langle\sigma_{z z}\right\rangle / \sigma^{m}\right\rangle 1\right)$. This reflects the fact that the particle phase carries the largest stresses due its largest volume fraction. The mean compressive stress is approximately 10 times lower than the tensile stress, and it declines with increasing $S$. In particular, its rapid decrease 
(a)

(b)
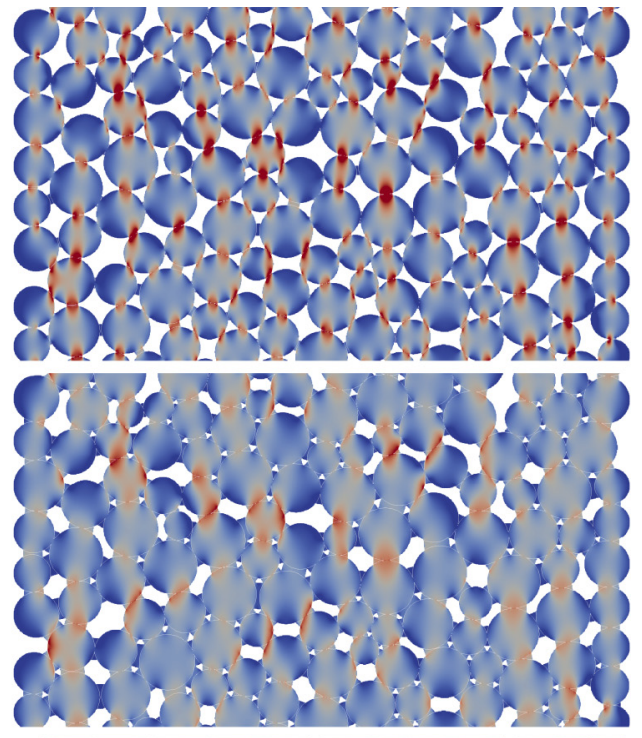

(c)

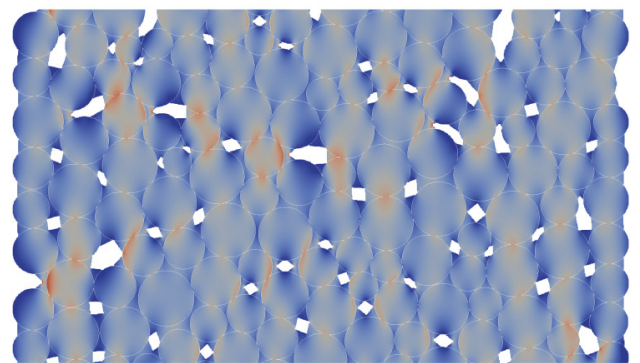

(d)

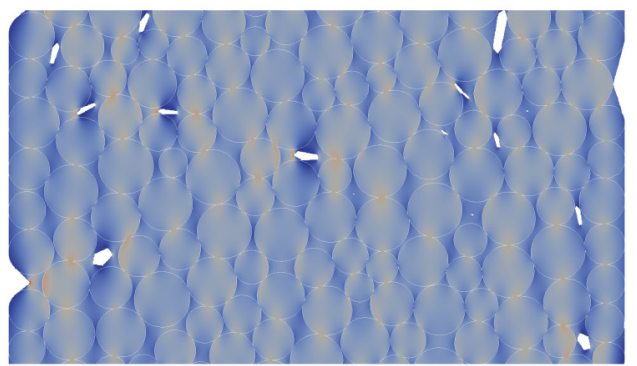

(e)
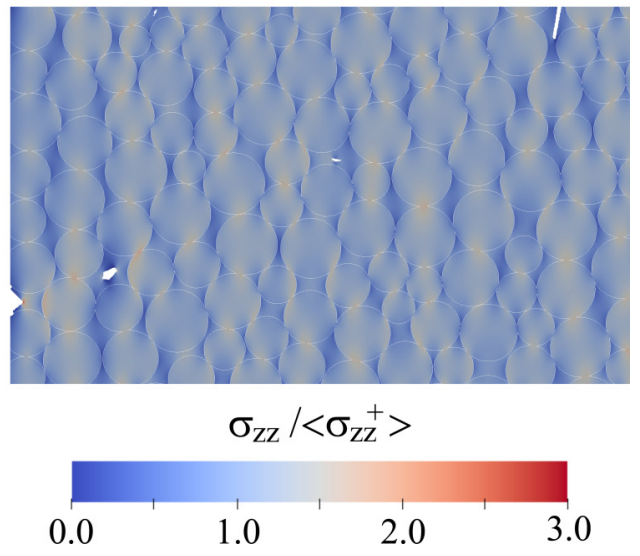

FIG. 15. Zooms in typical vertical stress maps in simulated aggregates for $\epsilon / \epsilon_{c}{ }^{m}=1.62 \times 10^{-2}$ and (a) $S=0.064$, (b) $S=$ 0.34 , (c) $S=0.67$, (d) $S=0.91$, and (e) $S=0.97$. The stress is normalized by the mean vertical tensile (positive) stress. The color scale ranges from heavy blue for zero stress to heavy red for strong stresses.

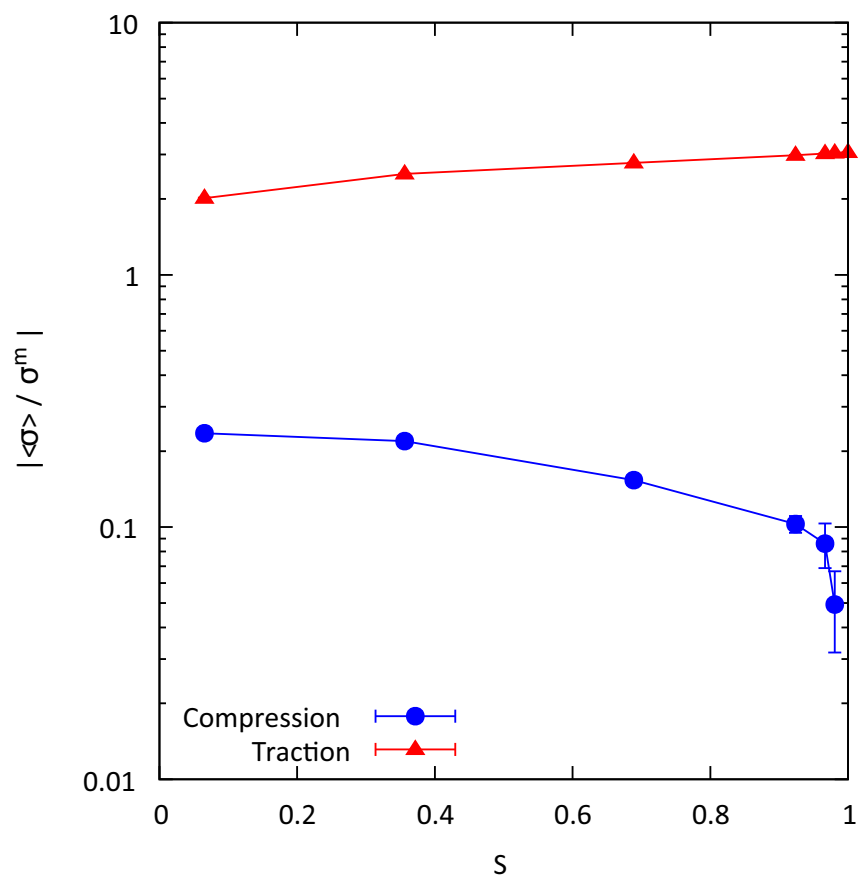

FIG. 16. Average value of tensile (positive) and compressive (negative) vertical stresses in the sample as a function of the matrix saturation factor $S$ for $\epsilon / \epsilon_{c}{ }^{m}=1.62 \times 10^{-2}$. The stresses are normalized by the stress in pure matrix at the same strain. The data points are averages over ten samples and the vertical error bars are the corresponding values of the standard deviation.

towards zero for values of $S$ close to 1 is a signature of load transfer from the particles to the matrix as the isolated voids are filled by the matrix.

The probability density functions (PDFs) of vertical stresses $\sigma_{z z}$ are shown in Fig. 17 for different values of $S$. For small values of $S$ we observe a broad distribution, with the largest tensile stresses extending to several times the average tensile stress of the sample. As $S$ increases the PDF shrinks, reflecting larger homogeneity of stress transmission. For all values of $S$ we observe a pronounced peak centered on the average tensile stress. Its increasing value with $S$ is another signature of the increasing homogeneity due to the pore-filling role of the matrix. The number of compressive stresses also declines and vanishes as $S \rightarrow 1$. The PDFs are also characterized by a peak or kink at zero stress. This peak is clearly a signature of the inhomogeneous stress distributions inside the particles. The strongest stresses are located at the contact zones between particles, but the stresses in the outer layers or rims of the particles that are not covered by the matrix are induced by the small deformations of the particles, and thus they are vanishingly small. These small stresses are also induced by the arching effect, i.e., deviation of stress lines due to local archlike structures, giving rise to low-stress regions [26].

A common feature of the stress PDFs is the exponential falloff at large tensile stresses:

$$
P(\sigma) \propto e^{-\theta \sigma_{z z} /\left\langle\sigma_{z z}^{+}\right\rangle}
$$




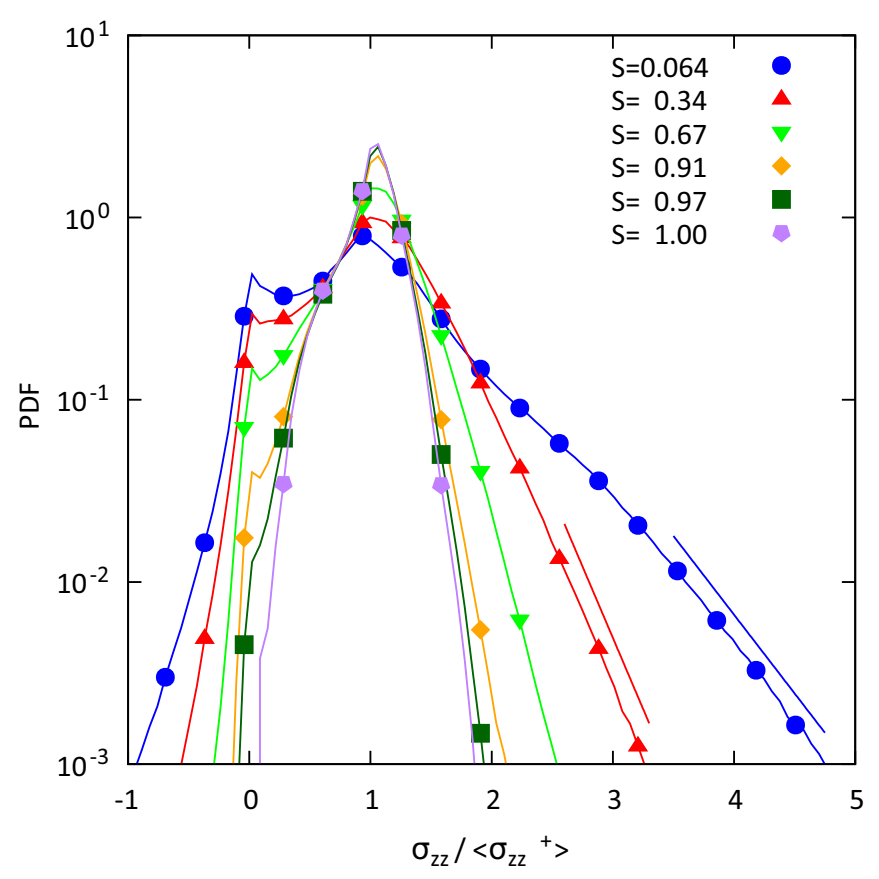

FIG. 17. Probability density function (PDF) of vertical stresses in granular aggregates for different values of $S$. The stresses are normalized by the average tensile stress (positive) $\sigma_{z z}^{+}$in each sample. On each curve, a symbol is drawn every five points. The straight lines represent exponential functions that are added here as a guide to the eyes.

This feature is a hallmark of force transmission in granular materials [26,59-62]. It is generally associated with the force chains, i.e., long-range correlations of strong forces across the contact network. Since we observe tensile stress chains (Fig. 15) in our aggregates, we may conclude that the exponential tails represent a signature of the contact network, connecting the particle phase at least in the gap-filling stage $(S<0.9)$. For larger values of $S$, the exponential tails can be attributed to disorder in the distribution of isolated pores [27]. This distinction between the two origins of the exponential tails is best quantified in Fig. 18, where the coefficient $\theta$ is plotted as a function of $S$. We observe a nearly linear increase of $\theta$ with $S$, followed by a rapid increase for the largest values of $S$. This sudden change is consistent with the rapid increase of the tensile strength in Fig. 14. This change can be described as a transition from stress concentration by the particle chains to stress concentration by pores.

\section{CONCLUSION}

The central idea of this work was to investigate the origins of the tensile strength in granular aggregates for a varying amounts of the binding matrix. The bond-based peridynamics model was used for its versatile nature, allowing for the discretization of both particle and matrix phases on the same underlying lattice and crack nucleation based on a local stress criterion. We showed that the Griffith model is in full agreement with peridynamics simulations for crack propagation, and we investigated the mesh effects and the choice of numerical parameters such as lattice step and the peridynamics

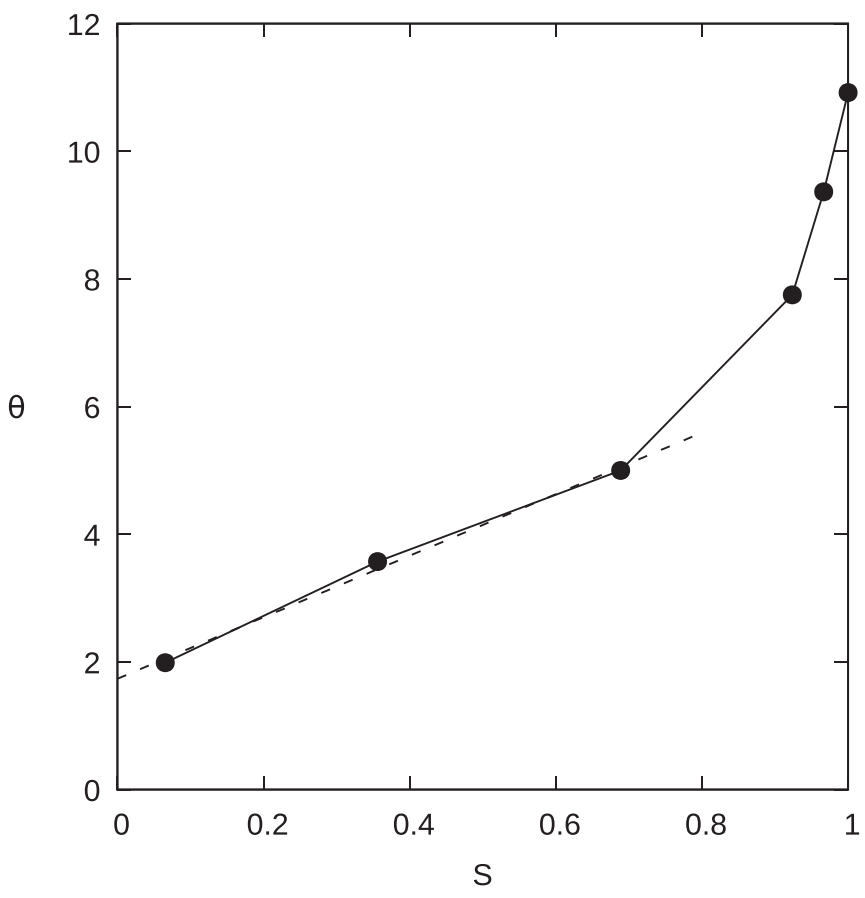

FIG. 18. The coefficient $\theta$ of the exponential falloff of tensile stresses as a function of the saturation factor $S$. The dashed line is plotted as a guide to the eyes.

horizon, identified here as the size of the fracture process zone.

The sample building procedure was designed to allow filling the gap space between pairs of particles by the binding matrix in the first stage. This procedure leads to a fraction $S=$ 0.9 of the interstitial space between particles to be filled. The remaining fraction is composed of isolated pores that are filled in a second stage. By subjecting the samples to tensile loading, we found that the contributions of the gap-filling matrix and pore-filling matrix to the tensile strength of the aggregates are nearly equal. The underlying physical effects are, respectively, tensile stress chains in the presence of a gap-filling matrix in the first stage and reduced stress concentration by the pores as they are filled by the matrix in the second stage.

The granular samples used in this work were dense packings created by compaction. Hence the number and volumes of isolated (higher-order) pores is small once all the gaps between pairs of particles are filled. For a lower packing fraction we expect this fraction to increase. It would therefore be interesting to consider in a future work samples of different packing fractions and evaluate the effect of the binding matrix. Further simulations can also be performed to analyze the shear strength of the aggregates by subjecting them to simple shear. A similar work in three dimensions (3D) requires much more computation time and memory. Although the pore structure in 3D packings is different, we still may distinguish the interparticle gap space from higher-order pores. For this reason, we expect similar results in 3D. Finally, the peridynamics approach can be used to investigate other classes of structured porous materials such as cellular solids where both porosity and stress correlations should underlie their strength and failure properties. 


\section{ACKNOWLEDGMENTS}

We are grateful to the INRAE MIGALE bioinformatics facility [63] and to the Genotoul Bioinformatics platform Toulouse Occitanie [64] for providing computing and storage resources.

\section{APPENDIX A: QUASISTATIC LOADING}

In order to enforce quasistatic loading, we damp elastic waves by adding a viscous force $-v \dot{\boldsymbol{u}}_{i}(t)$ to all material points [43]. The viscosity $v$ should be below the critical viscosity $\min \left\{\sqrt{2 m_{i} k}\right\}$ to avoid supercritical damping.

Upon failure, the stored elastic energy is released. Under stress-controlled conditions, this may lead to unstable crack propagation and bifurcations. To remain in quasistatic regime, one may decrease the loading rate at incipient breakage. Following the idea of the snap-back method [9], we use a regulation procedure which consists of applying the strain $\varepsilon$ at a rate $\Gamma(t)$ which depends on the cumulative damage energy $\omega:$

$$
\begin{gathered}
\dot{\omega}=-\frac{1}{\tau} \omega+\sum_{i} \delta(t-i \delta t) \Delta \omega_{i}, \\
\dot{\Gamma}=-\frac{1}{\tau}\left(\Gamma-\Gamma_{0} e^{-\frac{\omega}{\omega_{0}}}\right),
\end{gathered}
$$

where $\tau$ is a relaxation time, $\Gamma_{0}$ is the imposed strain rate, $\delta$ is the Dirac function, and $\Delta \omega_{i}$ represents the total damage energy of all bonds broken during the $i$ th time step. $\omega_{0}$ is the energy required to open a crack of length $\Delta \ell$ in mode I in the weakest phase. A value of $\Delta \ell$ below $1 \%$ of the system characteristic size is sufficiently small to ensure quasistatic loading with a reasonable CPU time. This value was used in all the simulations reported in this paper.

\section{APPENDIX B: PARALLEL IMPLEMENTATION}

Mechanical simulation of natural materials generally requires: (1) highly resolved samples accounting for their

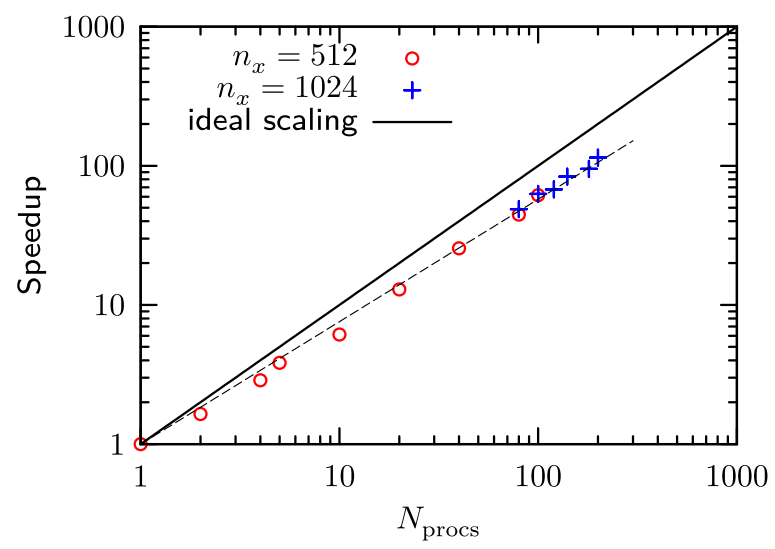

FIG. 19. Speedup of the developed peridynamics code (for $h / \delta x=3$ ) as a function of the number of processes for two resolutions: $512 \times 1024$ and $1024 \times 2048$.

complex microstructures, and (2) a large statistical set of tests to fully capture the mean behaviors and their variability. For these reasons and due to the CPU cost of the simulations, parallel computing is necessary.

We developed a parallel bond-based peridynamic computer code relying on the message-passing interface (MPI), which provides efficient routines to exchange data between a set of processes. In this code the spatial domain is split in equalsize subdomains which is attributed to a single process. The scalability of the code was tested on the French national cluster "Genotoul Bioinformatics." In this cluster, each computer node involves two IvyBridge 10 cores hyperthreaded microprocessors of frequency $2.5 \mathrm{GHz}$ interconnected through a Quad Data Rate (QDR) infiniband network for both MPI and input/output communications.

Figure 19 shows the speedup as a function of the number $P$ of processes involving up to 200 cores. The global trend shows a good scalability of the parallel algorithm. However, the speedup is not linear due to the nonlocal third-order neighborhood employed in the computation, which increases the exchange of data during the communication steps.
[1] H. J. Herrmann and S. Roux, Statistical Models for the Fracture of Disordered Media (Elsevier, New York, 2014).

[2] L. Dormieux, D. Kondo, and F.-J. Ulm, Microporomechanics (John Wiley \& Sons, New York, 2006).

[3] O. Buyukozturk and B. Hearing, Int. J. Solids Struct. 35, 4055 (1998).

[4] W. Gerstle, N. Sau, and S. A. Silling, Nucl. Eng. Des. 237, 1250 (2007).

[5] A. Manoylov, F. Borodich, and H. Evans, Proc. R. Soc. A 469, 20120689 (2013).

[6] L. Reig, C. Tojal, D. Busquets, and V. Amigó, Materials 6, 4868 (2013).

[7] R. Holtzman, Int. J. Numer. Anal. Methods Geomech. 36, 944 (2012).

[8] V. Topin, F. Radjai, J.-Y. Delenne, A. Sadoudi, and F. Mabille, J. Cereal Sci. 47, 347 (2008).
[9] E. Schlangen and J. van Mier, Cem. Concr. Compos. 14, 105 (1992).

[10] B. Chiaia, J. van Mier, and A. Vervuurt, Cem. Concr. Res. 28, 103 (1998).

[11] I. Merchant, D. Macphee, H. Chandler, and R. Henderson, Cem. Concr. Res. 31, 1873 (2001).

[12] M. A. Issa, M. A. Issa, M. S. Islam, and A. Chudnovsky, Eng. Fract. Mech. 70, 125 (2003)

[13] R. Affès, J.-Y. Delenne, Y. Monerie, F. Radjaï, and V. Topin, Eur. Phys. J. E 35, 117 (2012).

[14] R. Eckner, M. Krampf, C. Segel, and L. Krüger, Mech. Comp. Mater. 51, 707 (2016).

[15] J. Bauer, S. Hengsbach, I. Tesari, R. Schwaiger, and O. Kraft, Proc. Nat. Acad. Sci. 111, 2453 (2014).

[16] I.-H. Oh, N. Nomura, N. Masahashi, and S. Hanada, Scr. Mater. 49, 1197 (2003). 
[17] C. Zou, E. Zhang, M. Li, and S. Zeng, J. Mater. Sci. - Mater. Med. 19, 401 (2008).

[18] A. Fakhimi and A. Tarokh, Int. J. Rock Mech. Min. Sci. 60, 95 (2013).

[19] H. Laubie, F. Radjai, R. Pellenq, and F.-J. Ulm, J. Mech. Phys. Solids 105, 116 (2017).

[20] V. Topin, F. Radjai, J.-Y. Delenne, and F. Mabille, Powder Technol. 190, 215 (2009).

[21] S. Torquato, Random Heterogeneous Materials: Microstructure and Macroscopic Properties (Springer-Verlag, New York, 2002).

[22] S.-Y. Fu, X.-Q. Feng, B. Lauke, and Y.-W. Mai, Composites, Part B 39, 933 (2008).

[23] V. Topin, J.-Y. Delenne, F. Radjai, L. Brendel, and F. Mabille, Eur. Phys. J. E 23, 413 (2007).

[24] T. T. Wu, Int. J. Solids Struct. 2 , 1 (1966).

[25] F. Saadat, V. Birman, S. Thomopoulos, and G. M. Genin, J. Mech. Phys. Solids 82, 367 (2015).

[26] F. Radjai, D. E. Wolf, M. Jean, and J.-J. Moreau, Phys. Rev. Lett. 80, 61 (1998).

[27] H. Laubie, F. Radjai, R. Pellenq, and F.-J. Ulm, Phys. Rev. Lett. 119, 075501 (2017).

[28] T. Nguyen, J. Yvonnet, Q.-Z. Zhu, M. Bornert, and C. Chateau, Eng. Fract. Mech. 139, 18 (2015).

[29] K. Matouš, M. G. Geers, V. G. Kouznetsova, and A. Gillman, J. Comput. Phys. 330, 192 (2017).

[30] S. A. Silling, J. Mech. Phys. Solids 48, 175 (2000).

[31] S. A. Silling and E. Askari, Comput. Struct. 83, 1526 (2005).

[32] S. A. Silling, M. J. Epton, O. Weckner, J. Xu, and E. Askari, J. Elasticity 88, 151 (2007).

[33] Y. D. Ha and F. Bobaru, Int. J. Fract. 162, 229 (2010).

[34] Y. D. Ha and F. Bobaru, Eng. Fract. Mech. 78, 1156 (2011).

[35] E. Askari, F. Bobaru, R. B. Lehoucq, M. L. Parks, S. A. Silling, and O. Weckner, J. Phys.: Conf. Ser. 125, 012078 (2008).

[36] F. Bobaru, Model. Simul. Mater. Sci. Eng. 15, 397 (2007).

[37] E. Celik, I. Guven, and E. Madenci, Theor. Appl. Fract. Mech. 55, 185 (2011).

[38] A. Agwai, I. Guven, and E. Madenci, Microelectron. Reliab. 51, 2298 (2011).

[39] P. Perré, G. Almeida, M. Ayouz, and X. Frank, Ann. Forest Sci. 73, 147 (2016).

[40] J. Xu, A. Askari, and S. Silling, J. Aerosp. Eng. 21, 187 (2008).
[41] W. Hu, Y. Ha, and F. Bobaru, Int. J. Multiscale Comp. Eng. 9, 707 (2011).

[42] E. Oterkus and E. Madenci, J. Mech. Mater. Struct. 7, 45 (2012).

[43] B. Kilic and E. Madenci, Theor. Appl. Fract. Mech. 53, 194 (2010).

[44] S. Sun and V. Sundararaghavan, Int. J. Solids Struct. 51, 3350 (2014).

[45] F. Radjai and F. Dubois, Discrete Numerical Modeling of Granular Materials (Wiley ISTE, London, 2011).

[46] N. Sloane, A Handbook of Integer Sequences (Academic Press, New York, 1973).

[47] A. Freimanis and A. Paeglitis, Procedia Eng. 172, 284 (2017).

[48] A. Yaghoobi and M. G. Chorzepa, Comput. Struct. 161, 43 (2015).

[49] T. L. Anderson, Fracture Mechanics: Fundamentals and Applications (CRC Press, Boca Raton, FL, 2017).

[50] G. Irwin, Fracture Dynamics, Fracturing of Metals (American Society of Metals, Metals Park, OH, 1948).

[51] G. Irwin, J. Appl. Mech. 24, 351 (1957).

[52] A. P. Roberts and M. Teubner, Phys. Rev. E 51, 4141 (1995).

[53] J.-M. Gatt, Y. Monerie, D. Laux, and D. Baron, J. Nucl. Mater. 336, 145 (2005).

[54] P. Diehl, F. Franzelin, D. Pflüger, and G. Ganzenmüller, Int. J. Fract. 201, 157 (2016).

[55] C. Voivret, F. Radjai, J.-Y. Delenne, and M. S. El Youssoufi, Phys. Rev. E 76, 021301 (2007).

[56] E. Chichti, V. Lullien-Pellerin, M. George, F. Radjai, R. Affès, and J.-Y. Delenne, J. Food Eng. 190, 40 (2016).

[57] X. Frank, J.-Y. Delenne, and F. Radjai, EPJ Web Conf. 140, 02029 (2017).

[58] M. A. Issa, A. M. Hammad, and A. Chudnovsky, Int. J. Fract. 60, 97 (1993).

[59] N. Estrada, A. Taboada, and F. Radjai, Phys. Rev. E 78, 021301 (2008).

[60] F. Radjai, C. R. Phys. 16, 3 (2015).

[61] D. M. Mueth, H. M. Jaeger, and S. R. Nagel, Phys. Rev. E 57, 3164 (1998).

[62] T. S. Majmudar and R. P. Behringer, Nature (London) 435, 1079 (2005).

[63] MIGALE, INRAE, 2018. Migale Bioinformatics Facility, https: //doi.org/10.15454/1.5572390655343293E12.

[64] GenoToul Bioinfo, INRAE, 2018. GenoToul Bioinformatics Facility, https://doi.org/10.15454/1.5572369328961167E12. 